\title{
Status of the KLOE-2 Inner Tracker
}

\author{
Erika De Lucia ${ }^{1, \star}$ on behalf of the KLOE-2 Collaboration \\ ${ }^{1}$ Laboratori Nazionali di Frascati dell'INFN, via E. Fermi 40, 00044 Frascati (RM), Italy
}

\begin{abstract}
KLOE-2 at the DAФNE $\Phi$-factory is the main experiment of the INFN Laboratori Nazionali di Frascati (LNF) and is the first high-energy experiment using the GEM technology with a cylindrical geometry, a novel idea developed at LNF. Four concentric cylindrical triple-GEM detectors compose the Inner Tracker, inserted around the interaction region and before the inner wall of the pre-existing KLOE Drift Chamber to improve the resolution on decay vertices close to the interaction point. State-of-the-art solutions have been expressly developed or tuned for this project: single-mask GEM etching, multi-layer XV patterned readout, PEEK spacer grid, GASTONE front-end board, a custom 64-channel ASIC with digital output, and the Global Interface Board for data collection, with a configurable FPGA architecture and Gigabit Ethernet. Alignment and calibration of a cylindrical GEM detector was never done before and represents one of the challenging activities of the experiment. The Inner Tracker detector construction, operation, calibration and performance obtained with cosmic-ray muons and Bhabha scattering events will be reported.
\end{abstract}

\section{Introduction}

The KLOE-2 experiment at the INFN Laboratori Nazionali di Frascati (LNF) is the continuation of the KLOE experiment[1], upgraded with state of the art technology to improve its discovery potential. Data taking campaign started in November 2014 and will allow to pursue a physics program [2] including: i) CPT symmetry and quantum coherence tests with neutral kaons with an unprecedented precision [3], ii) high precision studies of $\gamma \gamma$-physics processes [4] and iii) search for new exotic particles that could constitute the dark matter [5,6]. The previous KLOE setup consisting of a huge Drift Chamber (DC) [7] and an Electromagnetic Calorimeter (EMC) [8], both immersed in a $0.5 \mathrm{~T}$ axial magnetic field, underwent several upgrades: i) state-of-the-art cylindrical GEM detector, the Inner Tracker (IT), to improve vertex reconstruction capabilities near the interaction region, ii) LET [9] and HET $[10,11] e^{+} e^{-}$taggers for $\gamma-\gamma$ physics, iii) CCALT and QCALT detectors [12], crystal and tile calorimeters positioned near the interaction point and along the beam-pipe with the goal of improving multi-photon detection in rare decays and the acceptance for rejecting $K_{L} \rightarrow 3 \pi^{0}$ background events in CP-violating $K_{L} \rightarrow 2 \pi^{0}$ decays respectively.

Starting from November 2014, KLOE-2 acquired $2.4 \mathrm{fb}^{-1}$ by July 2016 and restarted data taking in September 2016 with the aim of collecting more than $5 \mathrm{fb}^{-1}$ in the next 1-1.5 years [13].

KLOE-2 is the first high-energy experiment using the GEM technology with a cylindrical geometry: the IT was mounted on DAФNE beam pipe and inserted inside the KLOE apparatus in July 2013

${ }^{\star}$ e-mail: erika.delucia@lnf.infn.it 
and started its commissioning phase January 2014, defining its optimal working point and measuring its performance. Alignment and calibration of a cylindrical GEM detector was never done before and represents one of the challenging activities of the experiment.

\section{Detector description}

This innovative detector shown in Fig.1 is composed by four concentric cylindrical triple-GEM (CGEM) detectors with $70 \mathrm{~cm}$ total active length and at radii from $13 \mathrm{~cm}$, to preserve the $K_{S}-K_{L}$ quantum interference region, to $20.5 \mathrm{~cm}$ due to the constraints from DC inner wall. Each CGEM [14] is a triple-GEM detector composed by five concentric cylindrical electrodes (Fig.2): the cathode, to set the drift field, 3 GEM foils for the electron multiplication and the anode, acting also as readout circuit.

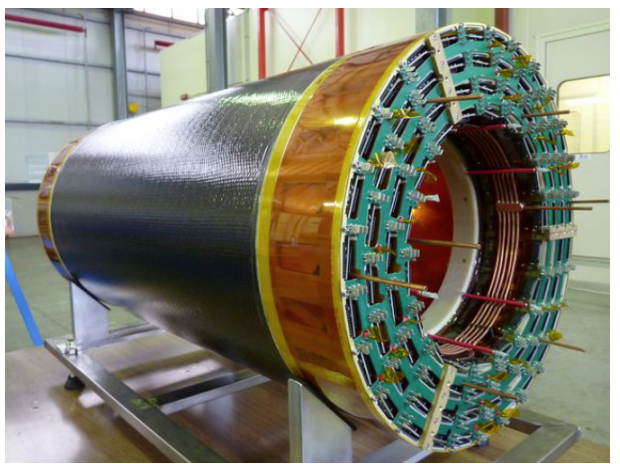

Figure 1. The Inner Tracker detector.

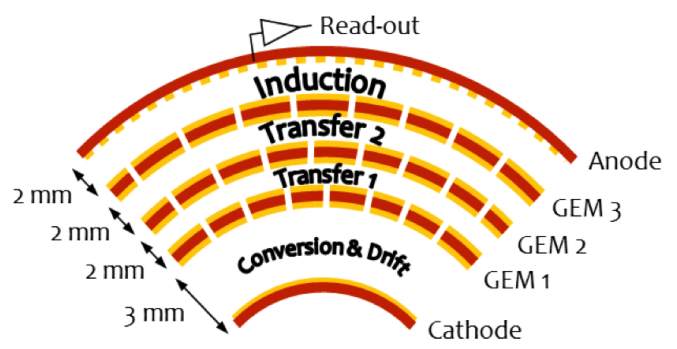

Figure 2. Cross-section of the triple-GEM detector.

The feasibility of a cylindrical triple-GEM detector with an XV readout was demonstrated during three years of R\&D [15-17]. These activities included the tuning and development of a new GEM manufacturing procedure of GEM foils of unprecedented size (up to $50 \times 100 \mathrm{~cm}^{2}$ ) with a singlemask electro-chemical etching of the micro-holes, produced with the TE-MPE-EM CERN group and followed and supported within the RD51 Collaboration [18]. The anode readout is patterned with longitudinal X strips with $650 \mu \mathrm{m}$ pitch, interleaved, on the same substrate and at the same level, with pads connected through internal vias to form V strips at an angle within $25^{\circ} \div 27^{\circ}$ and with $600 \mu \mathrm{m}$ pitch, for a total of about 30,000 FEE channels. Space coordinates are reconstructed using this dedicated XV strips patterned readout coupled to the GASTONE front-end, the 64 channel ASIC with digital output developed for the KLOE-2 experiment [19]. Data collection is then performed with a General Interface Board (GIB) with a configurable FPGA architecture, Gigabit Ethernet and the readout driver (ROD), also developed for KLOE-2 [20]. An $\mathrm{Ar} / \mathrm{iC}_{4} \mathrm{H}_{10}$ gas mixture was chosen, accounting for the most important operating parameters, including the discharge probability measured with $\alpha$-particles.

The IT has been inserted in the free space between the beam pipe and the inner wall of KLOE $\mathrm{DC}$ [7] providing $\simeq 150 \mu \mathrm{m}$ spatial resolution in the bending plane, $\sigma_{z} \simeq 2 \mathrm{~mm}$ along the beam line and $\simeq 6 \mathrm{~mm}$ on decay vertices close to the IP. The resolution on vertices close to the IP reconstructed from low-momentum charged secondaries is expected to improve of about a factor 2-3 [21]. 


\section{Detector Operation}

The construction of the KLOE-2 Inner Tracker was completed in March 2013 and in June 2013 this new sub-detector was integrated on DAФNE beam pipe and equipped with two cooling systems: copper coils as water radiators on the front-end electronics and air blowing on the IT end-caps and between the beam-pipe and the IT innermost layer. The Inner Tracker was finally inserted inside the KLOE-2 apparatus in July 2013.

The optimization of the IT operational parameters with colliding beams started in late 2014 during the first KLOE-2 data taking campaign, as a function of the $\mathrm{e}^{+} \mathrm{e}^{-}$beam currents and machine background conditions. Dedicated online monitoring procedures were developed and are currently used to check IT temperature, currents and voltages, together with occupancy and clustering performance [22] with DAФNE delivering collision data. To safely operate all KLOE-2 sub-detectors and for $\mathrm{e}^{+} \mathrm{e}^{-}$ injection optimization, the information from three indicators of the machine background level is sent online to DAФNE operators: the current measured on the first IT layer, the total current measured in the DC and the background level measured in the EMC end-caps. This allowed machine background conditions to be kept at an acceptable level having at the same time good collider uptime and luminosity performance. Then in order to measure efficiency and resolutions, dedicated procedures were developed exploiting the Drift Chamber track reconstruction and using cosmic-ray muon data and Bhabha scattering events.

Cosmic-ray muon tracks reconstructed in the Drift Chamber with $0.52 \mathrm{~T}$ magnetic field are used to evaluate IT efficiency. Tracks reconstructed in the DC are extrapolated to the Inner Tracker with straight-line approximation. The normalization sample for the efficiency evaluation is given by the events with two crossing points with each IT layer. We then look for reconstructed 3D clusters in the IT, close to expected position from the DC extrapolated track. High voltage scans have been performed setting different induction field values, namely $5 \mathrm{kV} / \mathrm{cm}$ and $6 \mathrm{kV} / \mathrm{cm}$. A single-view efficiency up to $98 \%$ has been measured by setting the induction field to $6 \mathrm{kV} / \mathrm{cm}$, as reported in Fig. 3 for Layer 1 , together with a satisfactory $95 \%$ two-view efficiency. All layers provide similar results. Presently

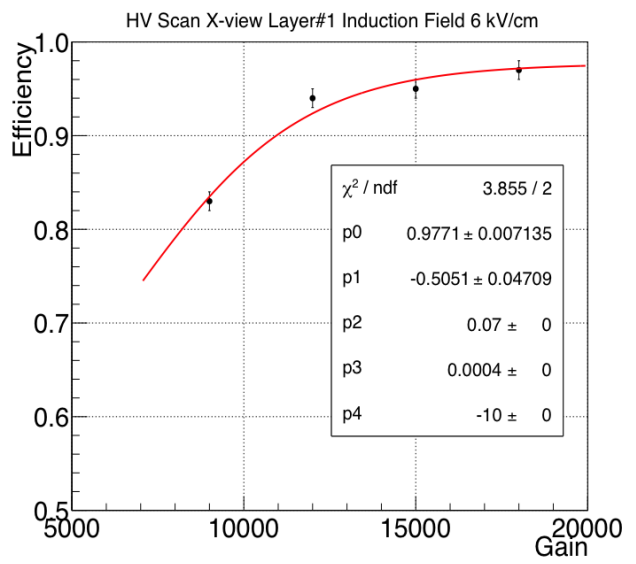

Figure 3. Layer 1 single-view efficiency as a function of the effective gain. Black points are experimental data, while the solid line is the Fermi-Dirac best fit curve.

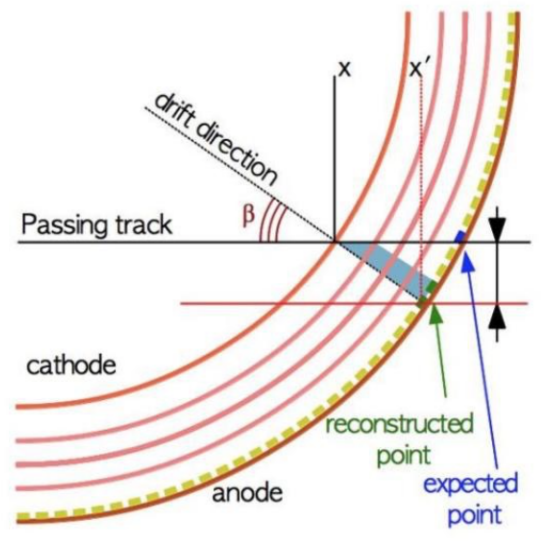

Figure 4. Non-radial tracks effect on the position measured with a cylindrical GEM prototype.

the IT is operated with a nominal gain of $1.2 \times 10^{4}$, with electric fields set at $1.5 / 3 / 3 / 6 \mathrm{kV} / \mathrm{cm}$ and 
GEM1/GEM2/GEM3 voltages set at 285/280/265 V. These values were chosen as a good compromise between detector efficiency and safe operation with collisions.

\subsection{Alignment and Calibration}

To properly perform track reconstruction using the IT information, alignment and calibration are first performed. During the R\&D phase of the project, two effects with $O(1-2 \mathrm{~mm})$ average corrections were observed and measured with planar XV prototypes at the test beams [21]. The first effect is related to non-radial tracks crossing the detector (Fig. 4): due to the angle between the electric field drift line direction and the incident track, the expected position will be shifted to the reconstructed point and the signal electron cloud will be spreaded. The second effect is due to magnetic field: the signal electron cloud experiencing the Lorentz force will be shifted and spreaded. According to the impact parameter of the track on the cylindrical detectors, these two effects will produces a focusing or de-focusing of the electron cloud. Cosmic-ray muon data have been used to evaluate the non-radial correction (without B-field) and the magnetic field influence (with B-field), and Bhabha scattering events to check both.

Alignment and calibration of the IT is performed with respect to DC reconstructed tracks, providing the expected position on each IT layer, and exploiting the residual distribution along the three coordinates. A first set of alignment and calibration parameters was obtained with cosmic-ray muons without B-field[23]. Before any alignment or calibration, the residual widths and mean values for Layer \#4 are: $\operatorname{Res}(\mathrm{x}) \sim 1.5 \mathrm{~mm}, \operatorname{Res}(\mathrm{y}) \sim 280 \mu \mathrm{m}, \operatorname{Res}(\mathrm{z}) \sim 3.6 \mathrm{~mm}$ and $\Delta x \sim 860 \mu \mathrm{m}, \Delta y \sim-50 \mu \mathrm{m}$ $\Delta z \sim 2 \mathrm{~mm}$. After the first alignment and calibration, residual distributions exhibit average widths along the $\mathrm{x}$-axis $\operatorname{Res}(\mathrm{x}) \sim 400 \mu \mathrm{m}$ for all layers and are all centered around zero within $50 \mu \mathrm{m}$. Results which are within expectations for the Inner Tracker resolution. Then starting from the B-field Off sample parameters, also a first set of calibration parameters for the cosmic-ray muon sample with B-field was obtained with average value $\operatorname{Res}(\mathrm{x}) \sim 550 \mu \mathrm{m}$ for all layers. Finally the average residuals have been cross-checked with Bhabha scattering events measuring $\operatorname{Res}(\mathrm{x}) \sim 400 \mu \mathrm{m}$ for all layers. Fig. 5 shows the path towards the first alignment and calibration results of the IT Layer \#4. This
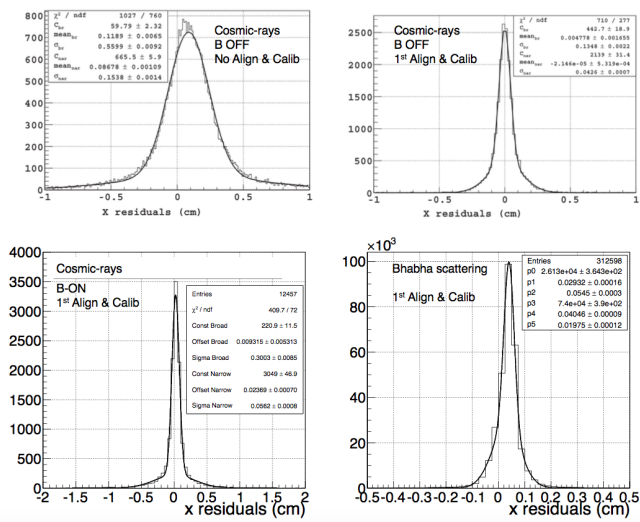

Figure 5. Layer \#4 residuals for the $\mathrm{x}$ coordinate: cosmic-ray muon B-field Off without (top left) and with (top right) alignment and calibration, cosmic-ray muon B-field On sample (bottom left) and Bhabha scattering events (bottom right).
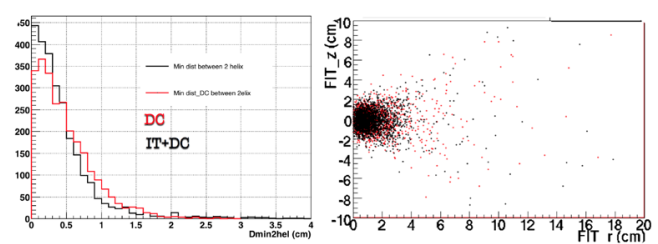

Figure 6. Very first comparison between simple vertex finder position obtained with DC (red) and IT+DC tracking (black) with two-pion Kaon decays. Left: two-helix distance. Right: simple vertex fit z position vs transverse radius 
validates our method and also suggests how to improve the alignment and calibration procedure in order to reach the best detector performance.

IT clusters are included in track reconstruction, using the Kalman filter technique and starting from DC hits and track parameters. Bhabha scattering events, selected as in the alignment and calibration procedure, are used as benchmark: i) alignment and calibration parameters obtained with the cosmicray muon sample are inserted, ii) then calibrated IT clusters are used for IT+DC integrated tracking.

First studies have been performed using both Bhabha scattering events and two-pion Kaon decays, and with a simple vertex finder based on minimum distance between extrapolated tracks, with DC only and using the integrated IT+DC tracking. A very preliminary comparison is shown in Fig. 6: left panel shows the two-helix minimum distance as obtained with a simple vertex finder using DC tracks only (red) and using the very first version of the integrated tracking IT+DC (black); in the right panel, with the same colour code, the $\mathrm{z}$ position vs transverse radius of the reconstructed vertex is shown. Presently we are setting up the official reconstruction with the IT+DC integrated tracking and its interface with the official vertex finder routine, tests both Bhabha scattering event and $K_{S} \rightarrow \pi^{+} \pi^{-}$ decays are ongoing.

\section{Conclusions}

KLOE-2 is the first high-energy physics experiment to build and operate a cylindrical GEM detector. Results reported are very positive and encouraging for the integrated tracking performance optimization phase and are also of extreme interest for the other experiments which will use cylindrical GEM detectors (BESIII) or are planning to (Novosibirsk).

The challenging task of aligning and calibrating this innovative detector was performed, with cosmic-ray muon data and Bhabha scattering events. Starting from Res(x) $\sim 1.5 \mathrm{~mm}$ width of the residual distribution with cosmic-ray muon B-field Off sample, the first alignment and calibration of the IT allowed $\operatorname{Res}(\mathrm{x}) \sim 400 \mu \mathrm{m}$ width to be reached, also with Bhabha scattering events. Further improvements are under study: we are presently working on refining the parameters from both the cosmic-ray muon B-field Off and On samples.

Alignment and calibration parameters obtained so far have been inserted in the IT+DC integrated tracking. First studies, performed using both Bhabha scattering events and two-pion kaon decays, show improvements already with the first and not yet refined alignment and calibration parameters. Detector reconstruction performance optimization phase started using both Bhabha scattering event and $K_{S} \rightarrow \pi^{+} \pi^{-}$decays for validation.

\section{Acknowledgements}

We warmly thank our former KLOE colleagues for the access to the data collected during the KLOE data taking campaign. We thank the DAФNE team for their efforts in maintaining low background running conditions and their collaboration during all data taking. We want to thank our technical staff: G.F. Fortugno and F. Sborzacchi for their dedication in ensuring efficient operation of the KLOE computing facilities; M. Anelli for his continuous attention to the gas system and detector safety; A. Balla, M. Gatta, G. Corradi and G. Papalino for electronics maintenance; C. Piscitelli for his help during major maintenance periods. This work was supported in part by the Polish National Science Centre through the Grants No. 2013/08/M/ST2/00323, 2013/11/B/ST2/04245, 2014/14/E/ST2/00262, 2014/12/S/ST2/00459. 


\section{References}

[1] Bossi F., De Lucia E., Franzini J. L., Miscetti S., Palutan M. and KLOE Collaboration, Nuovo Cimento, 30 (2008) 10.

[2] G. Amelino Camelia et al., Physics with the KLOE-2 experiment at the upgraded DAФNE, Eur. Phys. J. C 68619 (2010).

[3] E. Czerwinski for the KLOE-2 Collaboration, Overview of KLOE results on kaon physics and $K L O E-2$ perspectives, these proceedings

[4] S. Giovannella for the KLOE-2 Collaboration, KLOE results on hadron physics and perspectives for $K L O E-2$, these proceedings

[5] E. Graziani for the KLOE-2 Collaboration, Search for the dark boson at KLOE and perspectives for $K L O E-2$, these proceedings

[6] G. Venanzoni E. Graziani for the KLOE-2 Collaboration, KLOE results and perspectives for KLOE-2: running of alpha e.m., hadronic cross section, these proceedings

[7] M. Adinolfi et al., Nucl. Inst. \& Meth. A 488 (2002) 51.

[8] M. Adinolfi et al., Nucl. Inst. \& Meth. A 482 (2002) 51.

[9] Babusci D. et al., Acta Phys. Pol. B, 46 (2015) 87.

[10] Babusci D. et al., Acta Phys. Pol. B, 46 (2015) 81.

[11] D. Moricciani for the KLOE-2 Collaboration, Status of the KLOE-2 high energy taggers, these proceedings

[12] Happacher F. and Martini M., Acta Phys. Pol. B, 46 (2015) 87.

[13] D. Domenici for the KLOE-2 Collaboration, Status of the KLOE-2 experiment, these proceedings

[14] G. Bencivenni, D. Domenici, The Ultra-Light Cylindrical-GEM detector as Inner Tracker at KLOE-2, Nucl. Inst. \& Meth. A 581 (2007) 221.

[15] G. Bencivenni et al., A Time Domain Reflectometer with 100 ps precision implemented in a costeffective FPGA for the test of the KLOE-2 Inner Tracker readout anodes, Nucl. Inst. \& Meth. A 698 (2013) 185.

[16] A. Balla et al.,The cylindrical GEM detector for the KLOE-2 Inner Tracker, JINST 9 C01014 (2014).

[17] A. Balla et al., Status of the cylindrical-GEM project for the KLOE-2 inner tracker, Nucl. Inst. \& Meth. A 628 (2011) 18.

[18] M. Alfonsi et al., Activity of CERN and LNF groups on large area GEM detectors, Nucl. Inst. \& Meth. A 617 (2010) 151-154; presented at the $11^{\text {th }}$ Pisa Meeting on Advanced Detector, May 24-30, 2009, La biodola - Isola d'Elba (Italy).

[19] A. Balla et al., GASTONE64: A new front-end ASIC for the cylindrical GEM Inner Tracker of KLOE-2 experiment at DAФNE, Nucl. Inst. \& Meth. A 721 (2013) 523.

[20] P. Branchini et al., Front-end DAQ strategy and implementation for the KLOE-2 experiment, JINST 8 T04004 (2013).

[21] F. Archilli et al., KLOE-2 Collaboration, Technical design report of the inner tracker for the KLOE-2 experiment, arXiv:1002.2572v1 and LNF-10/3(P) INFN-LNF, Frascati,2010.

[22] A. Di Cicco, G. Morello, Commissioning of the Inner Tracker of the KLOE-2 experiment, Acta Phys. Polon. B 46 (2015) 73.

[23] A. Balla et al., The KLOE-2 Inner Tracker: Detector commissioning and operation, Nucl. Inst. \& Meth. A 845 (2017) 266. 